\title{
Time-Domain Simulation of Mixed Nonlinear Magnetic and Electronic Systems
}

\author{
A. D. Brown, Senior Member, IEEE, J. N. Ross, and K. G. Nichols
}

\begin{abstract}
This paper describes a technique for the simulation of complex magnetic systems intimately connected to any necessary drive electronics. The system is split into two Kirchhoffian domains, one magnetic and one electric. Two-way interaction between the domains is supported by a virtual device called a magnetoelectric differential gyrator. With this technique, arbitrarily complex, nonlinear, hysteretic magnetic systems may be simulated in the time domain, coupled to any appropriate nonlinear electronics, at a fraction of the cost of a comparable finite-element calculation. The capabilities of the system are demonstrated by the simulation of a feedback-controlled current-sensing system, and the simulation tracks the measured behavior of the system well outside its linear region, to the point that the nonlinear hysteretic core is being driven into and out of saturation, a consequence of a time delay inherent in the electronics. This is compared with a "conventional" electronic simulation of the same system, and the increased accuracy of this technique is clearly demonstrated.
\end{abstract}

Index Terms-Circuit simulation, magnetic circuits, magnetic components, nonlinear magnetic models.

\section{INTRODUCTION}

$\mathbf{S}$ IMULATION and modeling play a dominant role in the development of almost every high-technology electronic/electrical product today. The requirement of decreased time to market and the high cost of prototyping make "right first time" development of vital economic importance. Circuit and system simulation has been an established tool in the design and development of electronic systems for several decades.

The economics of the design and fabrication of high-performance ICs is well known; however, the design of power electronics circuits containing wound components is also extremely costly. Not only are there few accurate models available, but the manufacture of prototypes is itself an expensive and time-consuming process.

The accuracy of any simulation process can never be better than that of the component models used; much effort has been put into the production of more and more accurate models of semiconductor devices, of all varieties: RF, power, III-V, microwave and so on; magnetic devices have received relatively little input. The accuracy and availability of simulatable magnetic models, especially high power, remain low.

The concept of the duality between magnetic and electric circuits has been established for over half a century [1]-[6], and

\footnotetext{
Manuscript received December 21, 1998; revised May 25, 2000. This work was supported by ERA Technology Ltd., U.K., and Telcon Ltd., U.K.

The authors are with the Department of Electronics and Computer Science, University of Southampton, Hampshire SO17 1BJ, U.K.

Publisher Item Identifier S 0018-9464(01)00609-4.
}

the value in analyzing magnetic machines and systems where induced currents and fields are important has been clearly demonstrated.

The technique described in this paper hinges on a physical magnetic circuit being split into a number of discrete, lumped components joined at magnetic nodes. This is hardly contentious: an electrical circuit is normally analyzed in terms of lumped components instead of distributed fields. A spectrum of analysis methodologies exist: at one end lies finite-element analysis, which will provide the best possible accuracy, and take into account almost every possible electromagnetic effect. The computing cost is huge, making it extremely difficult to include any such model into any kind of transient analysis system [1]. At the other end of the spectrum, the equation for a linear inductor is one of the simplest to incorporate into a nonlinear simulator. In between, various electrical and phenomenological models exist [2], [3], [7] but these effectively break the magnetic component down into an electrical macromodel. The macromodel of [7], one of the most general, models a toroidal core with a single air gap, and incorporates leakage electrical inductance, wire electrical resistance, winding electrical capacitance, turns ratio, and coupling coefficients. The electrical inductance uses the Jiles-Atherton [4], [5] core model. Despite this level of complexity, it is not capable of delivering acceptable accuracy in certain circumstances [8], [9], because the model does not take into account winding or core geometries.

Electrical circuit simulators work by analyzing the behavior of electrical nodes, in terms of their potential (electromagnetic force) and the current (charge flux) that flows between them. These flows are controlled both by the node potentials and the electrical characteristics of the components joining them.

This paper describes an analysis technique based on a magnetic macromodel. In this macromodel, a magnetic circuit is described in terms of lumped parameter subcomponents, interconnected at "nodes" (cf. the electrical nodes of a lumped terminal transmission line macromodel). The macromodel may be embedded into electrical drive circuitry (the electromagnetic interactions are bidirectional) and analyzed by a circuit simulator in a manner similar to a purely electrical circuit. The terminal nodes of the macromodel are purely electrical, but an internal node may be electrical (potential is a representation of electromagnetic force, charge flux - current - flows between the nodes) or magnetic (potential is a representation of magnetomotive force, magnetic flux "flows" between the nodes). Thus, the concept of a topological circuit is extended to include magnetic circuit variables, allowing the accurate analysis of wound components with extremely complex geometries. The speed is comparable 
to a conventional electrical macromodel, but the analysis results reveal effects that are normally only visible using finite-element representations. Whilst the concept is not new [10], [11], the rapid decrease in cost of computing power over the last few years has made it timely to revisit the concept; it is now entirely feasible to connect highly complex electronic control systems to extensive magnetic models and simulate the overall structure in its entirety.

The focus of this paper is on the practical difficulties of simulating a mixed electronic/magnetic nonlinear system. Clearly, the accuracy of any system simulation will be a strong function of the accuracy of the primitive component models. However, there exists a vast and mature body of knowledge in the area already; for the purposes of this paper, the exact nature of the models employed is irrelevant-they may be replaced or enhanced easily.

Section II of the paper describes the lexicon of component primitives developed to support this analysis, and Section III contains details of numerical issues that have arisen during the course of this work. In its most abstract form, a continuouscircuit simulator "solves" a network, and no knowledge of the physical basis of the network is necessary. In practice, however, real simulators make judgments based on a priori knowledge of real electrical circuits (dynamic range of variable and time constants, for example), and it has been found that it is simply not possible to obtain sensible results without modifications to these (usually built in) assumptions.

Finally, Section IV provides examples of the use of this simulation technique. The agreement between experiment and simulation (using this technique) is extremely good; the agreement between experiment and the model of [7] is poor.

\section{The Primitive Components}

The primitive components used to model electrical circuits are well known, and their equations obvious: $V=i R, V=$ $L d i / d t$ and so on. The task of assembling a set of magnetic components is slightly more subtle, for three reasons:

- The absence of a magnetic "insulator" makes the identification of the functional terminals difficult (we note that finite-element analysis effectively sidesteps this problem by connecting everything to everything else).

- The practical absence of a magnetic monopole makes it difficult to conceptualize the idea of a charge quantum-extremely useful in a purely electronic simulator, although not strictly necessary.

- The difficulty of realizing an isolated "flux generator." However, electrical simulation has always utilized the ideal current source as an abstract component, even though this is as physically unrealizable as a magnetic flux generator.

It is noted in [12]: “... Both Laithwaite's [2] and Cherry's [6] treatments are in terms of circuits which are clearly defined physically, but this is not a necessary restriction if the equivalent circuit is viewed as a means of describing the characteristics of the device in terms of parameters referred to one or more pairs of magnetic 'terminals' ... ."
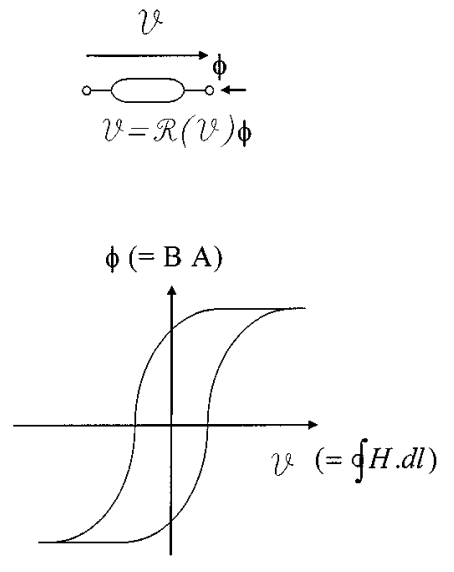

Fig. 1. Magnetic resistance.

\section{A. Dependent and Independent Variables}

The "across" and "through" variables in the electrical domain are voltage $(V)$ and current $(i)$, and the corresponding variables in the magnetic domain are $\operatorname{MMF}(\mathcal{V})$ and flux $(\varphi)$. It has been suggested [12] that $d \varphi / d t$, rather than $\varphi$, should be used for the latter. This is justifiable from a phenomenological viewpoint, and indeed sustainable algorithmically, but in practice, numerical errors (arising ultimately from the finite word length of software) mean that rounding errors will accumulate, eventually giving rise to the magnetic equivalent of a violation of charge conservation. Further, with a nonperiodic excitation and hysteretic primitive components, it is virtually impossible to establish a numerically sensible value for the implied constant of integration necessary to extract the flux. For these reasons, the compound circuit is solved in terms of $V, I, \mathcal{V}$, and $\varphi$.

Throughout the paper, the term "resistance" is used to refer to a two-terminal network component where the "across" and "through" variables are linearly related. "Inductance" refers to a two-terminal device where the "across" variable is linearly related to the time derivative of the "through" variable. "Capacitance" refers to a two-terminal network device where the "through" variable is linearly related to the time derivative of the "across" variable. To avoid confusion, each instance of each term is qualified with the appropriate domain name (electrical/magnetic).

\section{B. The Magnetic Resistance (Reluctance)}

Probably the best researched and yet conceptually simplest device, in its simplest form, this is a two-dimensional component that relates the flux flowing through it to the MMF across it-see Fig. 1. A wide variety of models have been published ([13]-[15], for example). For the purposes of this paper, the exact form chosen is irrelevant; our model is based on the model of Jiles-Atherton [4], [5]. In order to achieve a better fit with characteristics measured for real materials, both soft ferrites and high permeability soft steel, it has been found necessary to modify the original model by making the domain wall pinning parameter a decreasing function of the flux density $B$. An additional modification that has proved advantageous for some materials is to model the reluctance by a parallel combination of a hysteretic reluctance (using the Jiles-Atherton model) and 
TABLE I

GENERALIZING THE DOMAIN EQUIVALENCES

\begin{tabular}{|c|c|c|c|c|}
\hline Electrical domain & & & Magnetic domain & \\
\hline electrical resistance & $V=i R$ & $\Rightarrow$ & magnetic inductance -shorted turn & $\vartheta=\mathscr{L} \frac{d \varphi}{d t} ; \mathscr{L}=\frac{N^{2}}{R}$ \\
\hline $\begin{array}{l}\text { electrical } \\
\text { inductance }\end{array}$ & $V=L \frac{d i}{d t}$ & $\Rightarrow$ & $\begin{array}{c}\text { magnetic resistance (non- } \\
\text { hysteretic, linear reluctance) }\end{array}$ & $\ell=\Re \varphi(+k) ; R=\frac{N^{2}}{L}$ \\
\hline $\begin{array}{c}\text { electrical } \\
\text { capacitance }\end{array}$ & $i=C \frac{d V}{d t}$ & $\Rightarrow$ & & $\eta^{2}=N^{2} C \frac{d^{2} \varphi}{d t^{2}}$ \\
\hline
\end{tabular}

(a) From electrical to magnetic

\begin{tabular}{|c|l|l|l|l|}
\hline Magnetic domain & & Electrical domain & \\
\hline $\begin{array}{c}\text { magnetic resistance } \\
\text { (reluctance) }\end{array}$ & $\vartheta=\mathscr{R} \varphi$ & $\Rightarrow$ & electrical inductance & $V=L \frac{d i}{d t} ; L=\frac{N^{2}}{\mathscr{R}}$ \\
\hline magnetic inductance & $\vartheta=\mathscr{L} \frac{d \varphi}{d t}$ & $\Rightarrow$ & electrical resistance & $V=R i ; R=\frac{N^{2}}{\mathscr{E}}$ \\
\hline magnetic capacitance & $\varphi=\mathscr{\ell} \frac{d l^{0}}{d t}$ & $\Rightarrow$ & power source & $V=N^{2} \mathscr{C} \frac{d^{2} i}{d t^{2}}$ \\
\hline
\end{tabular}

(b) From magnetic to electrical

a nonlinear, but anhysteretic reluctance. In this way, it has been possible to obtain a good correlation between the measured and simulated characteristics of simple toroidal magnetic cores. The most serious problem in fitting the data with the model is that there are a large number of parameters, and as yet no general systematic method of fitting these has been found [16], [17].

\section{Sources}

Any network requires excitation (sources). For the magnetic network, these are the MMF (cf. voltage) and flux (cf. current). Constant sources may be used to represent permanent magnets (as opposed to hysteretic reluctance in a semipermanent magnetized state-the underlying principle is similar, but this representation is conceptually clearer).

A variation of the idea, consisting of a controlled electrical voltage source and a controlled MMF source, can be used to create the magnetoelectric differential gyrator. This asymmetric component forms the basis of the interface between the magnetic and electrical domains-see Fig. 2. (A demonstration of the consistency of this approach is given in Appendix A, which shows how a conventional electrical inductor may be realized using this technique.)

\section{The Magnetic Inductor}

The magnetic inductor (a two-terminal component in the magnetic domain with the characteristic equation $\mathcal{V}=\mathcal{L} d \varphi / d t$-see Table I) is the magnetic analog of a pure electrical resistance. It is a dissipative component, and plays a significant part in the modeling of eddy current losses

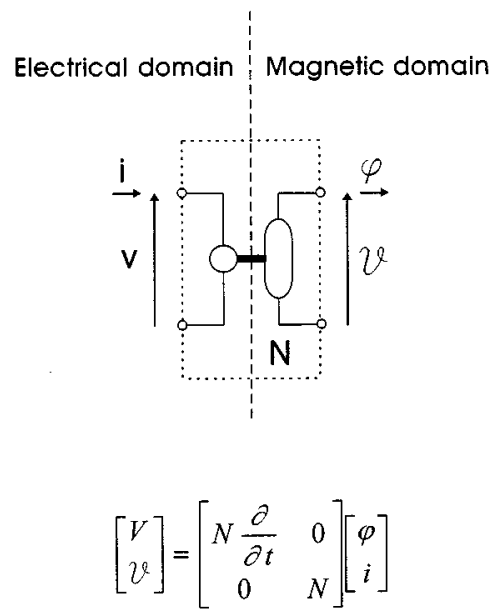

Fig. 2. The interface - a magnetoelectric differential gyrator (electrical inductance front end).

in Section IV. It is the magnetic domain equivalent of a shorted turn in the electric domain; analysis along the lines of that provided in Appendix B will provide a formal proof.

\section{E. The Hall Effect Device}

The model and equation for this are given in Fig. 3. The model is simplified in that it ignores high-frequency effects (for example, magnetic induction in the loop formed by the Hall voltage sensing circuit), but is adequate for our present purposes. 


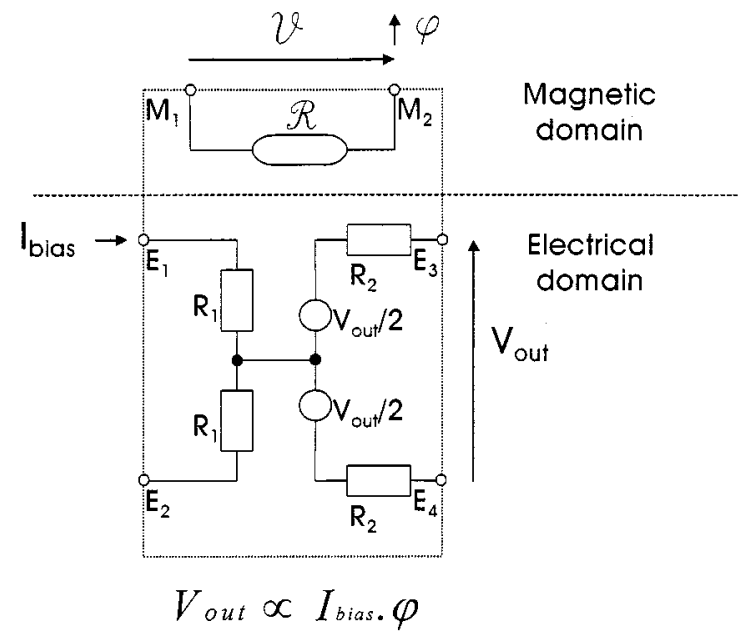

Fig. 3. Hall effect device.

(a) from electrical to magnetic

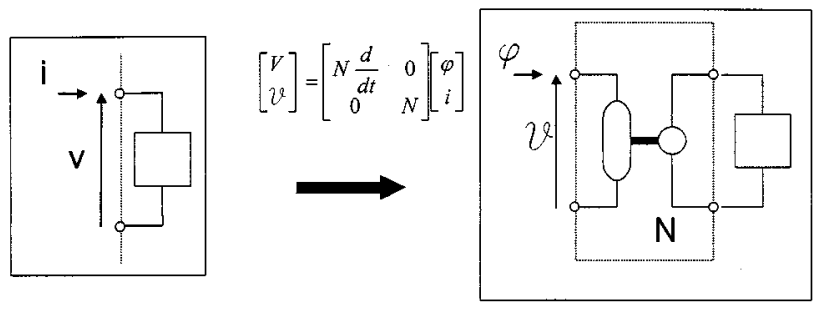

(b) from magnetic to electrical

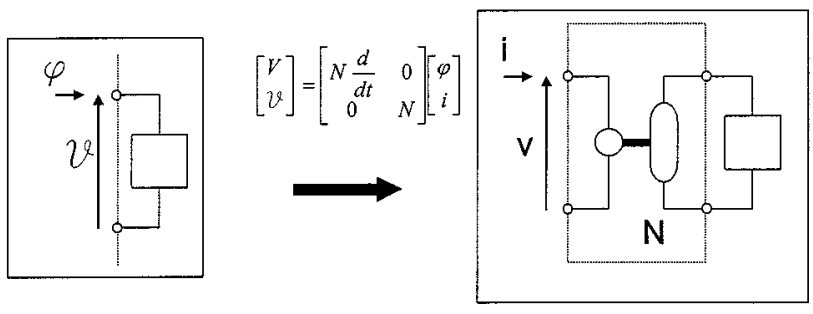

Fig. 4. Generalizing the domain equivalencies.

The device provides an interface between the two domains; information passes from the magnetic to the electrical domain. It has six terminals, two magnetic $\left(M_{1}\right.$ and $\left.M_{2}\right)$ and four electrical $\left(E_{1}-E_{4}\right)$. The two magnetic nodes are joined by a magnetic resistance $(R)$ and the others connect via the electrical domain network shown in the figure ( $\left.V_{\text {out }}, R_{1}, R_{2}\right)$. Ignoring the parasitic resistances $\left(R_{1}, R_{2}\right)$, the electrical voltage ("across" variable) asserted by the circuit across $E_{3}$ and $E_{4}$ is proportional to the product of the magnetic flux through the magnetic resistance $(R)$ and the electrical current flowing from $E_{1}$ to $E_{2}$.

\section{F. Generalizing the Domain Correspondences}

The gyrator allows us to "push" devices from domain to domain, as in Fig. 4; some components generated by the transformations are given in Table I. Some analogs are useful (for example, electrical inductance $\langle\Rightarrow$ magnetic resistance), whilst some appear mathematical curiosities; recall, however, that a component need not be useful (or even realizable) in isolation for it to be useful in a wider context. The magnetic capacitor, for example $(\varphi=\mathcal{C} d \mathcal{V} / d t)$, is hard to visualize physically, yet can play an important part in the simulation of magnetic circuits, as outlined in the next section.

\section{SimUlATION IMPLEMENTATION ISSUES}

The algorithms used in analog (electronic) circuit simulation are extremely numerically intensive, and a large amount of effort has been devoted to making commercial simulation engines "robust." This invariably takes the form of heuristic modifications that capitalize on a priori assumptions about "real" electronic circuits. Attempts to model mixed electronic and magnetic networks based on the network formalism described in this paper require the application of three specific modifications: these are described and justified in this section.

\section{A. Matrix Formulation}

Central to any march-in-time Newton-Raphson based nonlinear simulation is the construction of a Jacobian matrix [18], [19]. A number of representations are possible, but we use the modified nodal formulation

$$
\left[\begin{array}{c|c}
G & A \\
\hline-A^{T} & R
\end{array}\right]\left[\begin{array}{l}
V \\
I
\end{array}\right]=\left[\frac{I}{V}\right]
$$

where

$G \quad$ submatrix of conductive and capacitative terms;

$R \quad$ submatrix of resistive and inductive terms;

$A$ incident submatrix of branches onto nodes.

This is extended to accommodate magnetic terms

$$
\left[\begin{array}{c|c}
G, \mathcal{G} & A \\
\hline-A^{T} & R, \mathcal{R}
\end{array}\right]\left[\frac{V, \mathcal{V}}{I, \varphi}\right]=\left[\frac{I, \varphi}{V, \mathcal{V}}\right]
$$

where $\mathcal{G}$ and $\mathcal{R}$ are the magnetic permeance and reluctance terms.

$\{\mathcal{G}, \mathcal{R}, \mathcal{V}, \varphi\}$ are the variables of the algebra defining the magnetic domain; $\{G, R, I, V\}$ define the variables of the electrical-domain algebra. The two are interconnected by the constants of proportionality in the gyrators. The $[G, \mathcal{G}]$ submatrix is an amalgamation of the magnetic and electrical submatrices; the order of the entire system is increased to accommodate nodes from both domains. Numerical and algorithmic aspects of the overall solution process are covered in detail in [18], [19].

The simulation technique has reduced the entire system (both magnetic and electrical domains) to a discrete network. The behavior of the network is governed by the topology, plus the terminal characteristics of the components interconnecting the nodes; some of these components are controlled devices, i.e., their characteristics depend on the instantaneous values of the circuit variables elsewhere. (The gyrator introduced in Section II-F is an example of this.) The introduction of controlled components means that it is possible to create physical systems that reduce to disjoint networks.

The numerical inversion of the network equation (which can be necessary several times per simulation time step) requires that the matrix be nonsingular; this requirement, reflected back to the network generating the matrix, means (amongst other 


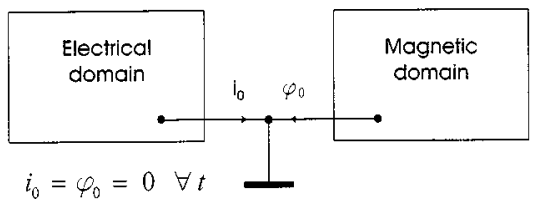

Fig. 5. Ensuring the Jacobian remains nonsingular.

things) that the network be nondisjoint, i.e., there must be some direct connection (aside from constants of proportionality and/or control functions) between the magnetic and electrical domains. The problem is overcome by making a single, direct "connection" between an electrical and magnetic node-see Fig. 5. Joining the two domains like this is physically defensible on the grounds that the current $i_{0}$ and flux $\varphi_{0}$ are always identically equal to zero: "through" variables must flow in loops, and there is only one connection between the two domains.

\section{B. Stability (1)}

A second issue is that of the stability of the solution process. Most conventional analog circuit simulators use the noniterative Gauss (or Gauss-Jordan or related) elimination method [20] to solve the linear set of equations that arise out of the nonlinear solution process at each time point in a simulation. Although it varies widely, typically, three solutions of the linear set are required at each time point of which there are perhaps a thousand or more in a simulation. Because time complexity of a solution of a linear equation set by standard methods is $O\left(n^{3}\right)$ ( $n$ is the number of variables), computational cost for larger circuits becomes prohibitive and so simulators exploit coefficient sparsity [21] of the set to reduce this complexity to $O\left(n^{1.1}\right.$ to 1.2$)$. This exploitation involves reordering equations and variables to minimize infill generation (nonzeros where previously there were zeros) during equation reduction.

A consequence of sparsity exploitation is the loss of any diagonal dominance of the coefficient matrix. This makes the practice of adding a small capacitance (i.e., a component where the "through" network variable is proportional to the rate of change of the "across" network variable) from every node to ground questionable (unless there is a realistic need for it). This adds a term $C / h$ to each diagonal element matrix, thus enhancing its diagonal dominance particularly with decreasing time step $h$. Only when any reordering leaves diagonal elements on the diagonal is the thesis valid. While this accelerates convergence of the iterative Gauss-Seidl and Gauss-Jacobi solution methods [20] used in relaxation simulators [22], the only reason it can help in conventional analog simulators is reduction of degree of node isolation.

In the magnetic domain, however, the technique of the last paragraph is not physically justifiable. The magnetic capacitor actually injects energy into the system (a simple proof is provided in Appendix B). Integrated over any time period, it will skew the results and render the simulation results increasingly inaccurate. As some simulators follow this practice without reference to the user, this problem is serious if one cannot very tightly control the process of simulation.

\section{Stability (2)}

Truly hysteretic components are not common in the electric domain (ferroelectric capacitors and rechargeable cells are examples; macrocircuits like Schmitt triggers are usually decomposed into a circuit with very high, but saturating, positive feedback, realized from nonhysteretic primitive components). When they do occur, the components are typically isolated from each other. Convergence problems derived from electrical hysteresis are unlikely to be encountered. In the magnetic domain, the situation is different.

The problem is associated with the change of hysteresis branch on the hysteresis loop that occurs when the magnetic field changes sense, i.e., at a cusp of the loop, and its interaction with the gyrator interface to the electric domain. The flux gradient with field is, in general, discontinuous, particularly below flux saturation.

Simulation in the magnetic domain describes the flux $\varphi$ (the through variable) as a nonlinear function of the MMF $\mathcal{V}$ (the across variable) and so the flux gradient of concern is $d \varphi / d \mathcal{V}$. Assume that the flux associated with this magnetic component flows through a gyrator which interfaces to the electric domain; the time rate of change of flux can then be expressed as

$$
d \varphi / d t=(d \varphi / d \mathcal{V}) \cdot(d \mathcal{V} / d t)
$$

and the emf induced across the electrical terminals of the gyrator is

$$
V=N \cdot(d \varphi / d t)=N \cdot(d \varphi / d \mathcal{V}) \cdot(d \mathcal{V} / d t) .
$$

Now, at the cusp $d \varphi / d \mathcal{V}$ can change abruptly but, in reality, at that point $d \mathcal{V} / d t$ must be zero because $\mathcal{V}$ is changing sense (monoticity) so the induced voltage $V$ is zero. Further, there is no discontinuity in $V$ because the approach to zero is smooth on one branch of the hysteresis loop and the departure from zero is smooth on the other branch.

Unfortunately, analog simulation is a crude approximation to reality in which continuous time is discretized into a sequence of time points, and gradients (with respect to time) are estimated with finite-difference expressions. Consequently, there is no guarantee that the estimation of $d V / d t$ will be zero at the cusp and so, as $d \varphi / d \mathcal{V}$ changes abruptly on passing the cusp, there can be an abrupt change in the value of the induced voltage $V$.

In conventional simulators, abrupt changes of a circuit variable greater than a local-truncation-error (LTE) limit are trapped and cause recalculation with a smaller time step, repeatedly, until the LTE limit is satisfied (this is the precision control system [23] which compares the solution with that of the previous time point). This, however, is clearly unlikely to work in the situation above (cusp passing) and so the simulation fails because of equation ill-conditioning (the time step is effectively cut to zero). Note, however, the situation does not affect the nonlinear solution process because convergence of this is independent of previous time-point data.

A solution to this problem is to disable all LTE checking on the solution subvector corresponding to electrical nets connected to terminals of (magnetic) gyrators. In all simulators of 
which we are aware this is not possible without code changes; further, although internal LTE checking of all nodes is made, LTE checking of branch currents is carried out only for nonlinear components, and the gyrator is a linear component.

In the simulator we are using, LTE checking of the voltages of nets connected to the gyrator has been disabled and replaced by LTE checking of the appropriate electrical branch currents. We have encountered no problems of simulation failure with this strategy, even when the electrical sides of a number of gyrators are in series, but their magnetic domains are separate from each other (magnetically isolated electrical inductances in series).

\section{SimULATION EXAMPLES}

\section{A. Modeling Eddy Current Losses}

Here we examine a model for a high-permeability steel, tapewound, toroidal core, such as is used in current transformers. The core to be modeled is wound with Mumetal tape, $0.1 \mathrm{~mm}$ thick and $7 \mathrm{~mm}$ wide, giving a total cross-sectional area of 16.6 $\mathrm{mm}^{2}$ and a mean magnetic path length of $61 \mathrm{~mm}$. The hysteresis properties of the core were measured at low frequency $(0.3 \mathrm{~Hz})$ - see Fig. 6(a); at frequencies above a few hertz the distortion of the hysteresis curve by the eddy currents is noticeable. The hysteresis curve was used to generate the reluctance model for the core material.

The eddy current loss model for the core is generated by assuming that the cross section of each lamination in the core can be split into concentric regions, each carrying a flux perpendicular to the section and a current sheet circulating around the cross section-see Fig. 6(b).

If we now assume that a) the model for each lamination is the same, and b) the excitation is the same, without loss of generality we may identify the corresponding nodes in each subcircuit, and create a single $\mathcal{L}-\mathcal{R}$ ladder magnetic equivalent circuit for the entire toroid. (Recall that the reason for using laminations is to significantly decrease the electrical conductivity of the core.)

This approach is equivalent to modeling the eddy current losses using a single $R-L$ ladder network in the electric domain [24], [25]. A linear core model using a six-section ladder is chosen as a compromise between computational efficiency and accuracy. This idea is analyzed in detail in [26]. Following [25], the cross-section segments have been assumed to decrease in thickness moving from the center of the lamination to the surface; the cross-sectional areas of successive segments decrease in a geometric series. Thus, the value of the reluctance elements, which are inversely proportional to the area, will also follow this geometric series. The magnetic inductance is equal to the inverse of the electrical resistance experienced by the eddy current circulating around each element. For thin, wide laminations both the area of the segment and the electrical resistance seen by the circulating current are proportional to the thickness of the segment, and so the magnetic inductance also varies inversely with the area of the segments.

To extract the parameters for the circuit of the core model, linear small-signal simulation was used alongside practical measurements to deduce $\mathcal{L}_{0}$ and $\mathcal{R}_{0}$ (Fig. 7). Measurements (a)

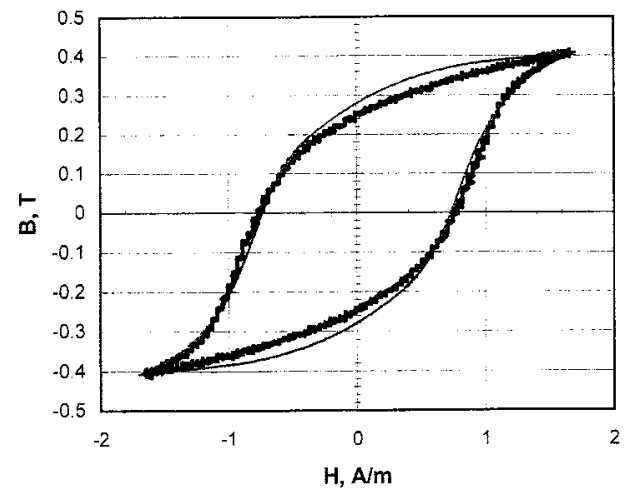

Fitted curve used to generate reluctance model

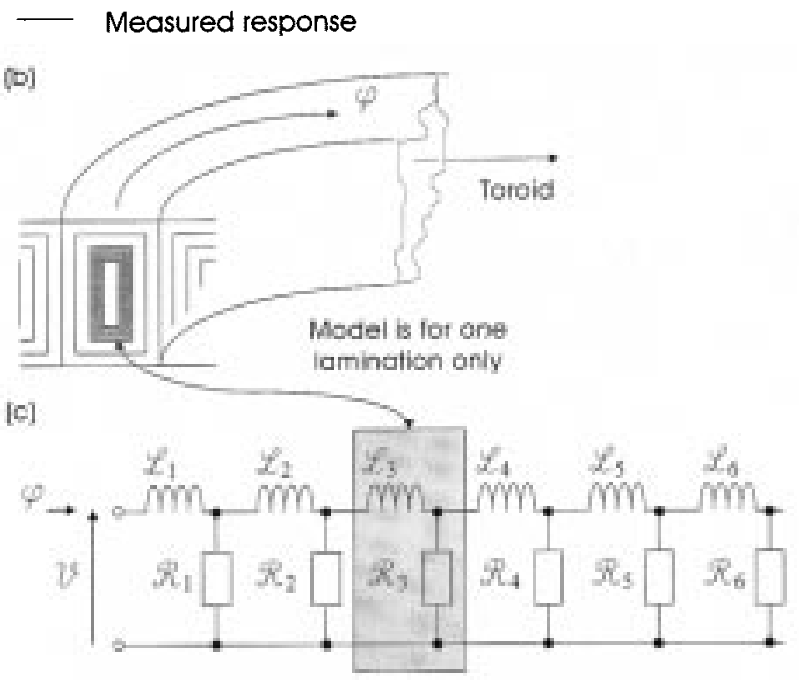

\begin{tabular}{|c|c|c|c|}
\hline$\partial_{0}=18 \Omega^{\prime}$ & & $K_{n}=$ & \\
\hline$Z_{1}=$ & $x_{0} * 0.101$ & $\Re_{1}=$ & $x_{b} / 0.101$ \\
\hline$x_{2}=$ & $x_{0}=0.121$ & $r_{3}=$ & $x_{0} / 0.121$ \\
\hline$y_{3}=$ & $\mathscr{x}_{0}=0.145$ & $r_{3}=$ & $\vartheta_{2} / 0.145$ \\
\hline$X_{4}=$ & $L_{0} \cdot 0.175$ & $\Re_{4}=$ & $9_{0} / 0.175$ \\
\hline$I_{1}=$ & $\mathscr{L}_{0} \cdot 0.209$ & $R_{3}=$ & $x_{0} / 0.209$ \\
\hline $1_{t}=$ & $L_{0} \cdot 0.251$ & $r_{1}=$ & $x_{0} / 0.251$ \\
\hline
\end{tabular}

$\mathscr{L}_{i}=\mathscr{L}_{0} \mathrm{k} ; \quad \Re_{1}=\Re_{0} \mathrm{k} ; \quad \mathrm{k}=1.2$

Fig. 6. Toroid core

were made on a core wound with a primary winding of 10 turns and a secondary winding of 100 turns. The primary was excited with a constant current of $0.5 \mathrm{~mA}$ (peak) at frequencies between $20 \mathrm{~Hz}$ and $50 \mathrm{kHz}$ and the in-phase and quadrature components of the secondary voltage were measured (data points in Fig. 7). The in-phase component at $20 \mathrm{~Hz}$ was too small to be measured.

In order to extend the simulation to include a nonlinear core model, the hysteresis loop for the core was measured at a frequency of $1 \mathrm{~Hz}$ [Fig. 6(a)]. To drive the system into nonlinearity, an excitation of $1.6 \mathrm{Am}^{-1}$ is used here. These data, together with the measured small-signal, low-frequency reluctance (derived from Fig. 7) were used to derive the parameters required by the Jiles-Atherton model for the nonlinear core. 

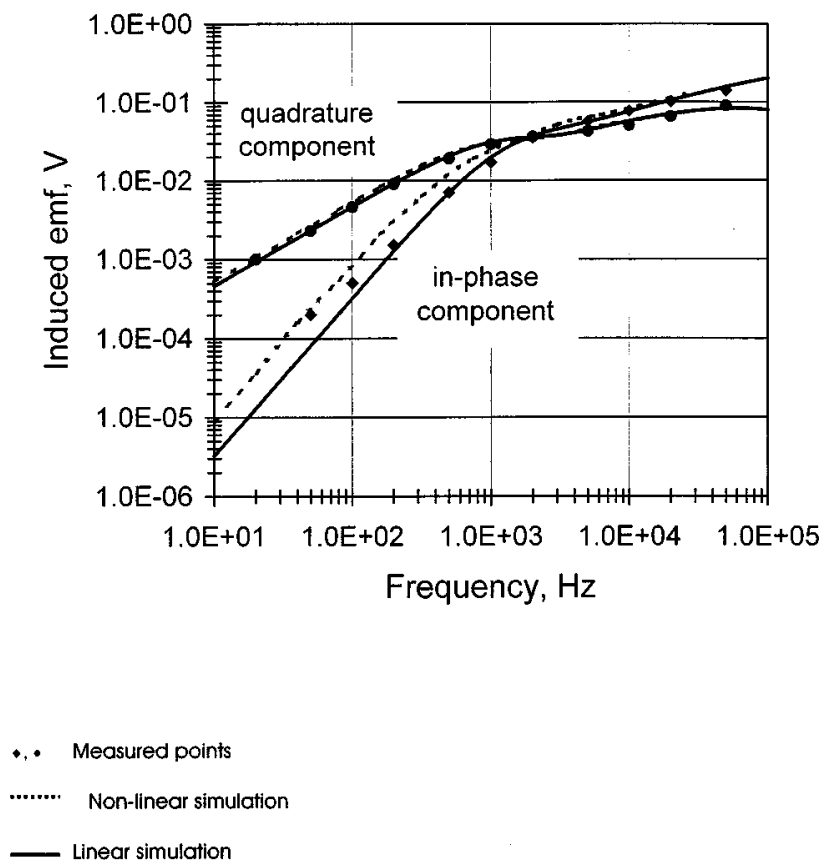

Fig. 7. Eddy current loss simulation and experiment.

The results of both the linear and nonlinear simulations are shown in Fig. 7, together with the measurements used to deduce the parameters. As expected, the linear model predicts a lower in-phase (loss) component than the measured values at low frequencies. The nonlinear model predicts a value that is too large. The most probable explanation for this excess loss is that when the nonlinear core model is set to give good agreement for major loops [as in Fig. 6(a)] it tends to predict minor loops which are more open (give greater loss) than those measured.

\section{B. Current Sensor}

Building on the previous example, we now use the technique to simulate the operation of a flux-nulling current transducer. A conventional current transformer is modified by cutting the core and inserting a Hall sensor to measure the core flux. This is then used with an amplifier to supply current to the secondary of the transformer, nulling the flux (Fig. 8). At low frequency the secondary current is determined by the feedback loop, while at high frequency the current is determined by the normal transformer action. A significant improvement in performance at high frequency is possible by only partially cutting the core, leaving a "magnetic short" across the gap containing the flux sensor. To a good approximation this does not reduce the flux in the gap (for a given MMF) but increases the total flux. This improves the performance of the current transformer as a passive device by reducing flux leakage while not significantly reducing the performance of the flux-nulling circuit. The amplifier used in the circuit is a wideband operational amplifier followed by a simple class B booster stage to drive sufficient current into the secondary. The amplifier must have a low output impedance at high frequency to enable the current transformer to operate satisfactorily as a passive device at high frequency.

The circuit simulated used a toroidal ferrite core (3E2 ferrite) with a magnetic path length of $57 \mathrm{~mm}$ and a core cross section

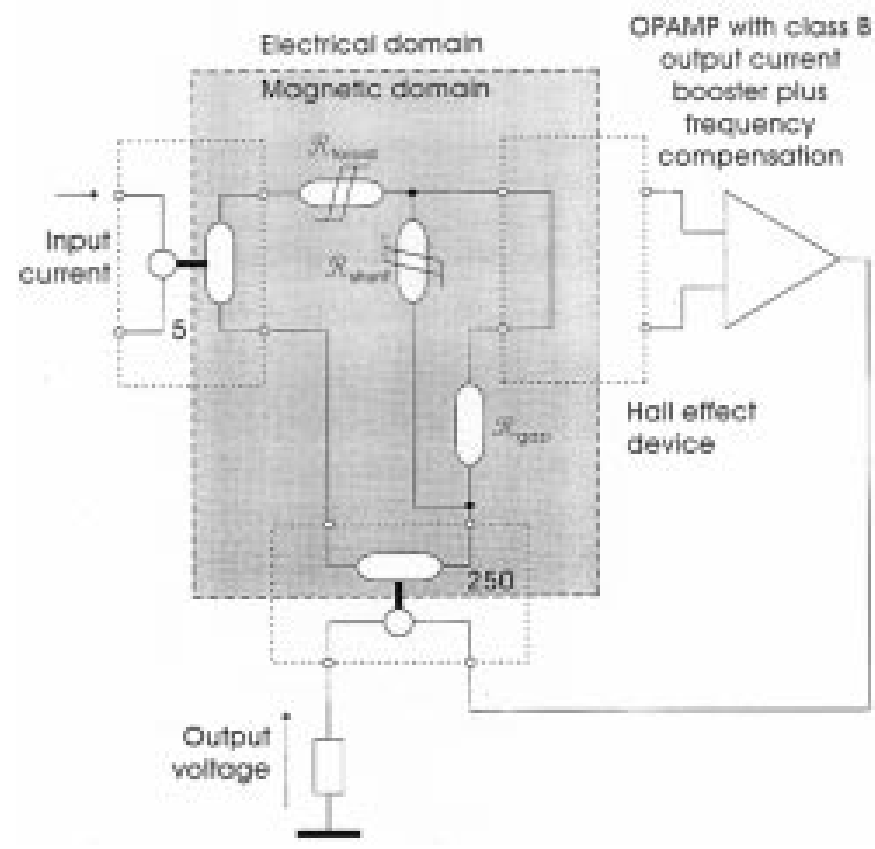

Fig. 8. Equivalent circuit of the current sensing system.

of $31.5 \mathrm{~mm}^{2}$. The core is partially cut with a $1-\mathrm{mm}$ slot to accommodate the Hall element leaving a magnetic short with an area of about $1 \mathrm{~mm}^{2}$. The magnetic properties of the core were measured by applying a sinusoidal voltage of amplitude $5 \mathrm{~V}$ to the secondary winding of 200 turns and measuring the current waveform. The core with its winding was then simulated. The parameters for the model for the $3 \mathrm{E} 2$ ferrite had previously been found from measurements on an uncut toroid. The cut toroid was modeled as a hysteretic reluctance for the main body of the toroid, a linear reluctance for the air gap, and a nonlinear reluctance for the magnetic short across the gap. The effective length and cross section of the magnetic short were adjusted so that a reasonable fit was obtained with the measured behavior of the core and a simulation of the experiment. The result of overlaying the experimental and simulated values is shown in Fig. 9.

With a wideband operational amplifier the current transducer provides a flat frequency response up to a frequency of much greater than $100 \mathrm{kHz}$, with a good step response. In order to illustrate the nonlinear modeling of the core, a slower amplifier (LM741) was used. With this amplifier there is a significant dip in the frequency response of the current transducer as the system transfers from operating as a flux-nulling device to a conventional current transformer. In this transfer region the flux in the core may build up to a value that leads to saturation of the magnetic short, as illustrated in Fig. 10 where the simulated and measured responses of the current transformer to a square wave current are shown. The large dip in the response after the rising edge is associated with saturation of the flux in the magnetic short. The simulated values for the flux in the magnetic short and the flux in the gap are shown in Fig. 11. The flux in the magnetic short saturates while the flux in the gap rises sharply shortly after the direction of the source current is reversed. It is apparent that the flux in the gap is nulled by the action of the feedback circuit after the initial transient. However, the flux in the magnetic short, and the total core flux, are nonzero because 


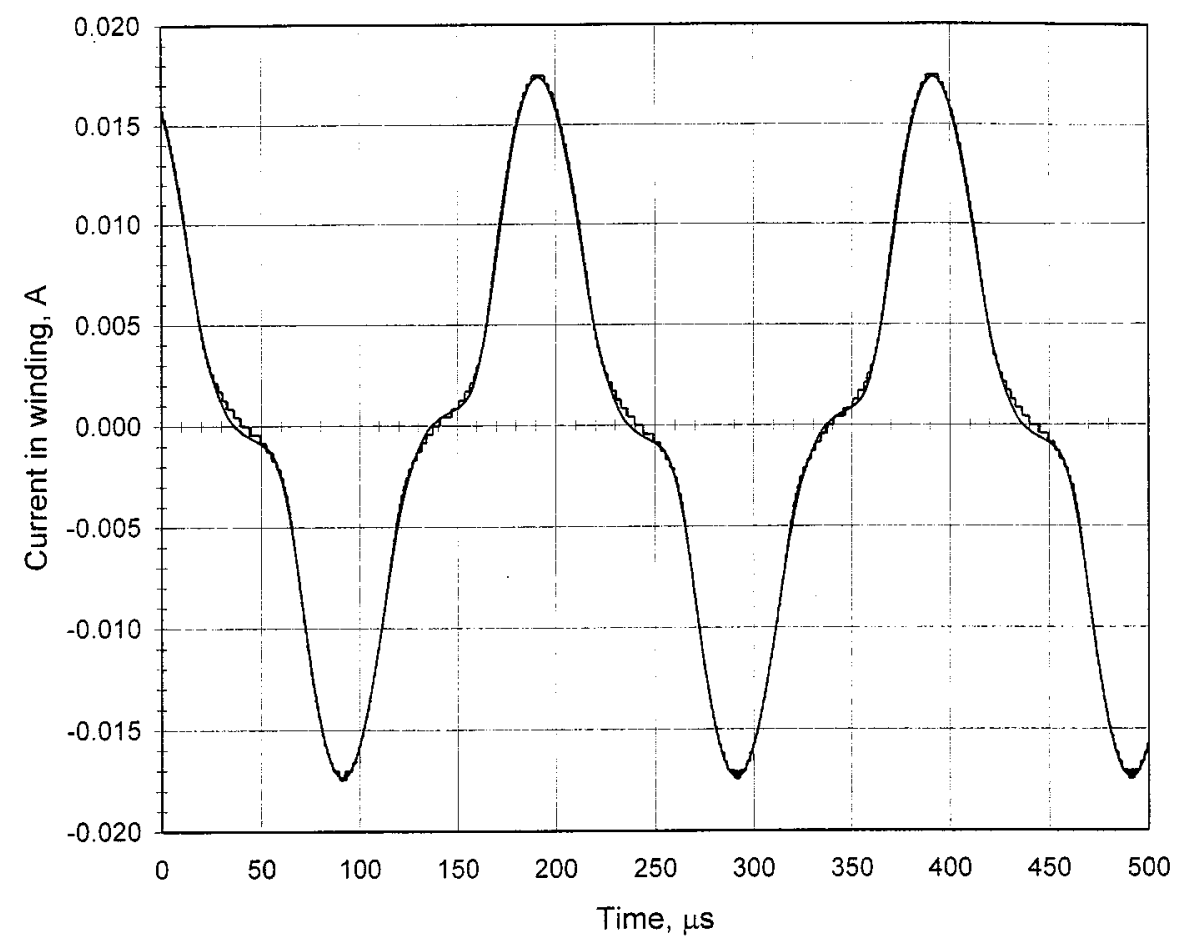

Fig. 9. Experimental and simulated current traces for the nonlinear toroid.

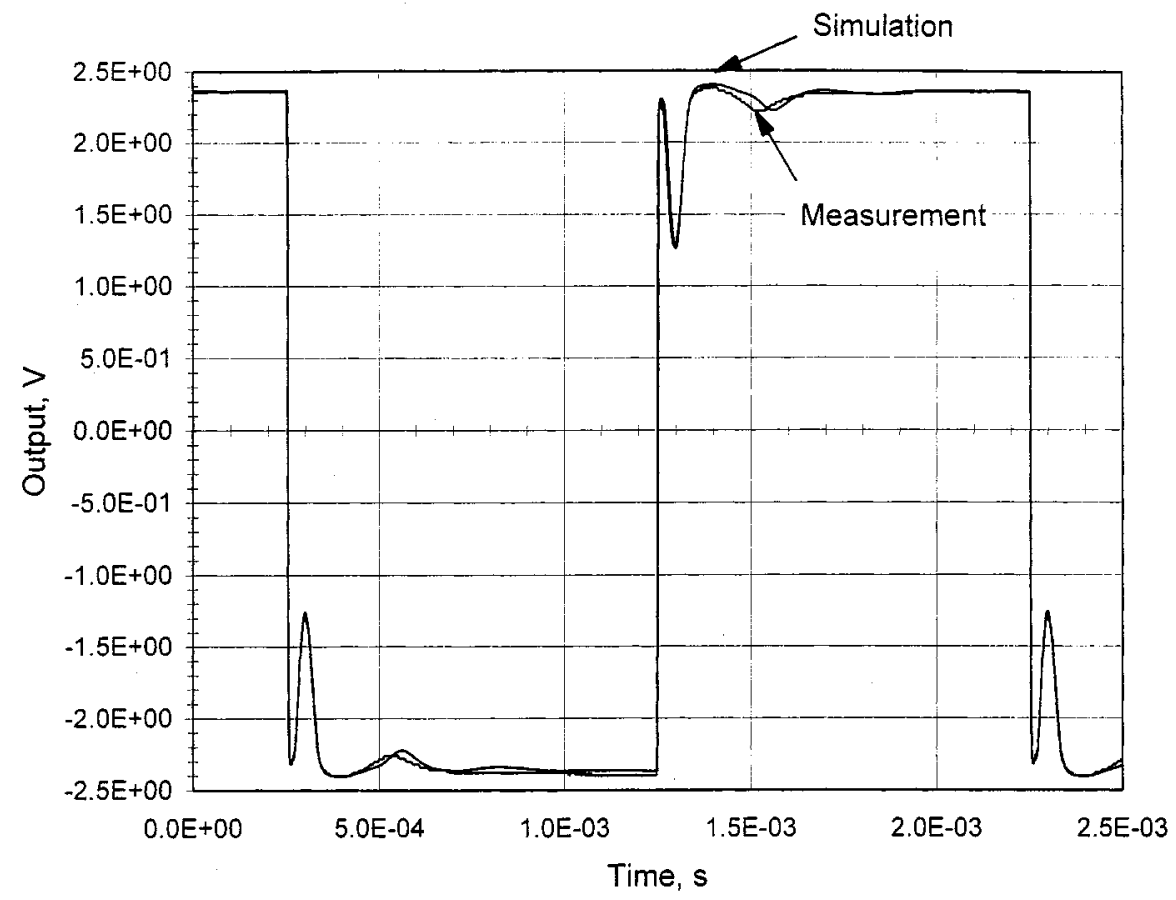

Fig. 10. Flux saturation in the magnetic shunt.

of the hysteresis of the magnetic short. The residual magnetization in the core when the gap flux has been nulled results in a small but significant error in the secondary current. The net MMF in the core is identical in form to the flux in the gap shown in Fig. 11 (the two are proportional). After the initial transient it falls to a low value (about $0.045 \mathrm{~A}$ ). This represents the difference between the ampere-turns for the primary and secondary windings, which are each of the order of $10 \mathrm{~A}$. Thus, the residual error at low frequency between the primary and secondary currents is of the order of $0.45 \%$. (The experimental residual error could not be measured with sufficient accuracy to verify this prediction.) The residual error is only about $0.12 \%$ if the simulation is performed with a linear magnetic shunt. If the amplifier is replaced by a faster operational amplifier, the core will not be driven into saturation; however, the shunt may still retain residual magnetization, which could be changed by a transient 


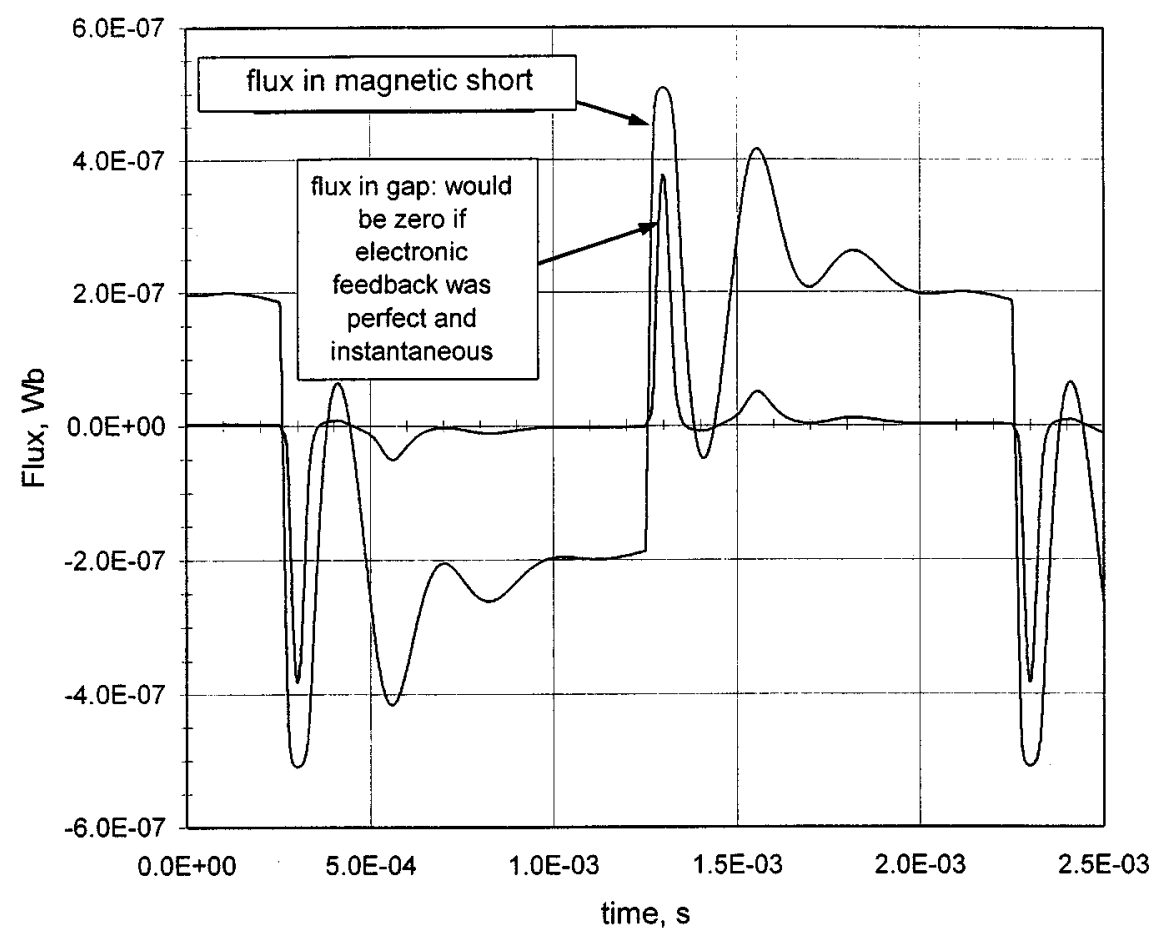

Fig. 11. Flux time histories in the shunt and the air gap. Note that the net MMF in the toroid is proportional to the flux in the gap.

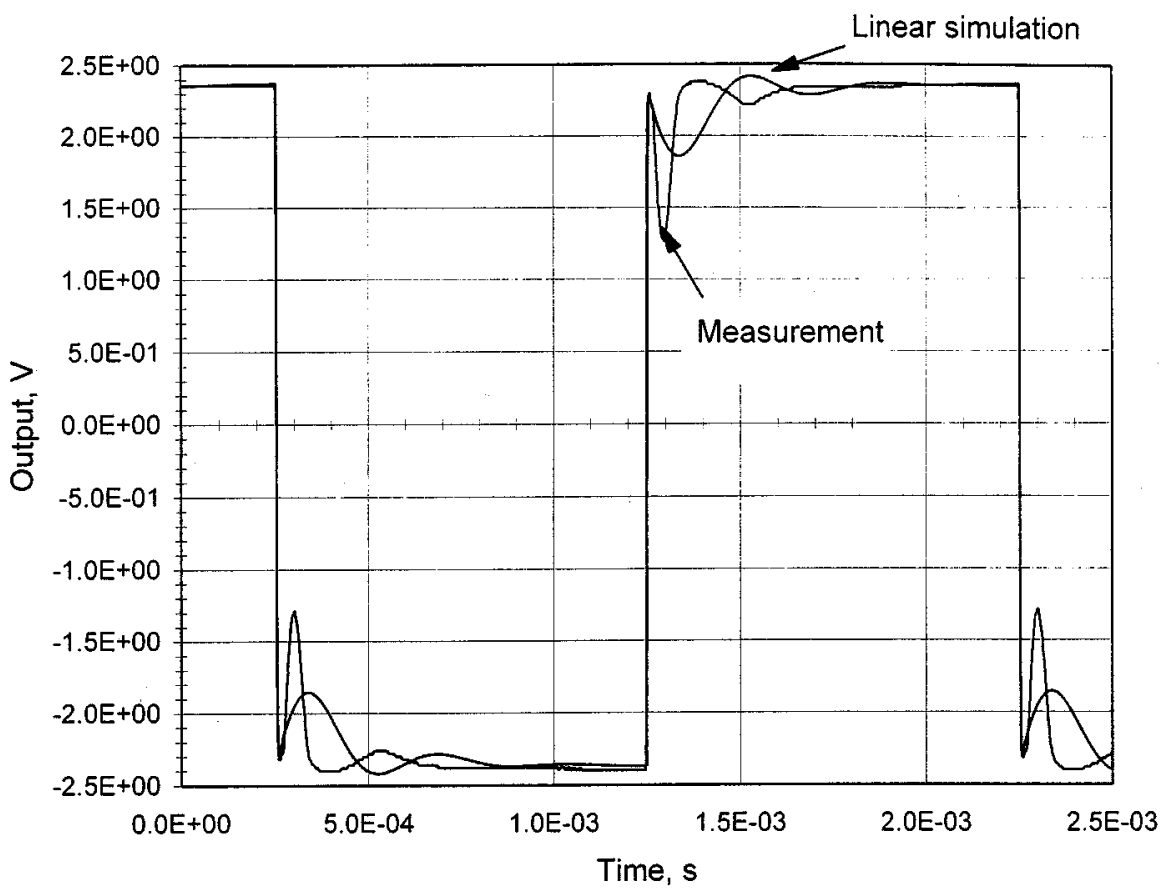

Fig. 12. Simulation of Fig. 10 performed entirely in the electrical domain.

overload. In this case the residual magnetization would result in an offset which will change if the residual magnetization is changed.

Finally, the results of Fig. 10 should be compared with Fig. 12, which depicts the best simulation results that could be obtained from a linear electrical model; attempts to reproduce the results using the PSPICE built-in Jiles-Atherton model [4], [5] fail because: i) the flux variable is not accessible;

ii) multisegment cores are not supported; and

iii) leakage electrical inductance is not directly supported.

\section{FinAl COMMENTS}

A simulation technique has been developed and demonstrated to allow mixed magnetic and electrical systems to be analyzed 


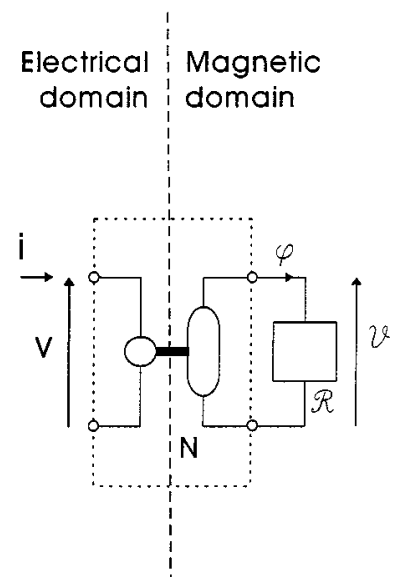

Fig. 13. Synthesis of an electrical inductance from magnetic components.

in the time domain, where components from each of the domains are treated on an equal footing. Using this technique, the agreement between experimental measurement and simulation is excellent, whereas the results of simulating entirely in the electrical domain are less accurate. The simulation times of example 2 are typically less than $1 \mathrm{~min}$, on a single-processor PIII machine. (A time-dependent finite-element analysis requires a complete static finite-element analysis every time step—see Section III-A—and will typically take two orders of magnitude longer to run.)

We note finally that it is not sufficient to simply create magnetic models and blindly "plug them in," to a third-party electrical simulation engine, as various elements of the simulator must be (slightly) modified before satisfactory solutions can be obtained. This means that access to the simulation engine source is required. Alleviating this requirement will be the subject of further work.

\section{APPENDIX A}

\section{THE SyNTHESIS OF AN ELECTRICAL INDUCTANCE FROM MAGNETIC COMPONENTS}

Consider Fig. 13: The interface between the two domains is realized by the gyrator, and the flux loop generated by the electrical winding is closed by the reluctance $\mathcal{R}$, in this case a composite assumed to represent the reluctance of the flux loop passing through the core plus any associated air gaps.

From the equation in Fig. 2, we have

$$
\left[\begin{array}{l}
V \\
\mathcal{V}
\end{array}\right]=\left[\begin{array}{cc}
N \frac{\partial}{\partial t} & 0 \\
0 & N
\end{array}\right]\left[\begin{array}{l}
\varphi \\
i
\end{array}\right]
$$

and $\mathcal{V}=\mathcal{R} \varphi$.

Eliminating both $\mathcal{V}$ and $\varphi$ from these equations and rearranging gives us

$$
V=\frac{N^{2}}{\mathcal{R}} \frac{d i}{d t}
$$

the familiar electrical inductance equation, with $L \equiv N^{2} / \mathcal{R}$. Any nonlinearity in $L$ is generated by an appropriate nonlinearity in $\mathcal{R}$.

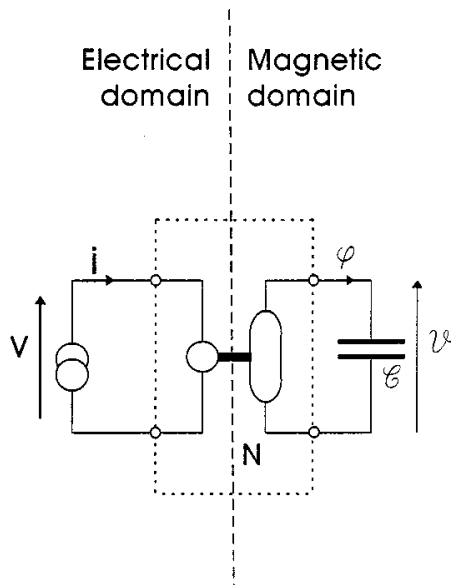

Fig. 14. Magnetic capacitor as a power source.

\section{APPENDIX B}

The Magnetic CAPacitor as a Power Source

That the magnetic capacitor generates power can be easily demonstrated: The characteristic equation of such a device is

$$
\phi=\mathcal{C} \frac{d \mathcal{V}}{d t} .
$$

This can be gyrated back to the electrical domain (i.e., take $\delta / \delta t$ of each side and note that $\mathcal{V}=N i$ ) to give

$$
V=N^{2} \mathcal{C} \frac{d^{2} i}{d t^{2}} \quad \text { (from Fig. 4). }
$$

We now inject current into the device; without loss of generality, choose a sine wave, $i_{0} \sin \omega t$ (see Fig. 14). Then $V(t)=-N^{2} \mathcal{C} i_{0} \omega^{2} \sin \omega t$ and the energy dissipated by the gyrated magnetic capacitor in one complete cycle is given by

$$
\begin{aligned}
U & =\int_{0}^{2 \pi} i(t) v(t) d t \\
& =-N^{2} \mathcal{C} i_{0}^{2} \omega^{2} \int_{0}^{2 \pi} \sin ^{2} \omega t d t \quad<0 .
\end{aligned}
$$

If a component dissipates negative energy, it must be an energy source.

\section{REFERENCES}

[1] H. C. Lai, D. J. Leonard, D. Rodgers, and N. Allen, "Dynamic simulation of electric machines coupled to external circuits using finite elements," Stud. Appl. Electromagn. Mater., Suppl. Int. J. Appl. Electromagn. Mater, vol. 6, pp. 407-410, 1995.

[2] E. Laithwaite, "Magnetic equivalent circuits for electrical machines," Proc. Inst. Electr. Eng., vol. 114, no. 11, pp. 1805-1809, 1967.

[3] W. Dierking and C. T. Kleiner, "Phenomenological magnetic core model for circuit analysis programs," IEEE Trans. Magn., vol. MAG-8, pp. 594-596, Sept. 1972.

[4] D. C. Jiles and D. L. Atherton, "Theory of ferromagnetic hysteresis," $J$. Magn. Magn. Mater, vol. 61, pp. 48-68, 1986.

[5] - "Ferromagnetic hysteresis," IEEE Trans. Magn., vol. MAG-19, pp. 2183-2185, Sept. 1983.

[6] C. Cherry, "The duality between interlinked electric and magnetic circuits and the formation of transformer equivalent circuits," Proc. Phys. Soc. B, vol. 62, pp. 101-111, 1949.

[7] J. H. Chan and A. Vladimirescu et al., "Nonlinear transformer model for circuit simulation," IEEE Trans. Computer-Aided Design, vol. 10, pp. 476-482, Apr. 1991. 
[8] D. A. G. Pedder, A. D. Brown, and J. A. Skinner, "A contactless electrical energy transmission system," IEEE Trans. Ind. Electron., vol. 46, pp. 23-30, Feb. 1999

[9] S. C. Wong and A. D. Brown, "Analysis, modeling and simulation of series-parallel resonant converter circuits," IEEE Trans. Power Electron., vol. 10, pp. 605-614, Sept. 1995.

[10] C. T. Kleiner, "Application of a phenomenological core model in the SYSCAP circuit analysis program," IEEE Trans. Magn., vol. MAG-19, pp. 2186-2188, Sept. 1983.

[11] D. Nitzan, "MTRAC: Computer program for transient analysis of circuits including magnetic cores," IEEE Trans. Magn., vol. MAG-5, pp. 524-532, Sept. 1969.

[12] C. J. Carpenter, "Magnetic equivalent circuits," Proc. Inst. Electr. Eng., vol. 115 , no. 10 , pp. $1503-1511,1968$.

[13] M. L. Hogdon, "Applications of a theory of ferromagnetic hysteresis," IEEE Trans. Magn., vol. 24, pp. 218-221, Jan. 1988.

[14] K. H. Carpenter, "A differential equation approach to minor loops in the Jiles-Atherton hysteresis model," IEEE Trans. Magn., vol. 27, pp. 4404-4406, Nov. 1991 .

[15] K. H. Carpenter and S. Warren, "A wide bandwidth, dynamic hysteresis model for magnetization in soft ferrites," IEEE Trans. Magn., vol. 28, pp. 2037-2040, Sept. 1992.

[16] S. Prigozy, "PSPICE computer modeling of hysteresis effects," IEEE Trans. Educ., vol. 36, pp. 2-5, Feb. 1993.

[17] D. C. Jiles and J. B. Thoelke, "Theory of ferromagnetic hysteresis: Determination of model parameters from experimental hysteresis loops," IEEE Trans. Magn., vol. 25, pp. 3928-3930, Sept. 1989.
[18] V. Litovski and M. Zwolinski, VLSI Circuit Simulation and Optimization. London, U.K.: Chapman \& Hall, 1997.

[19] C. W. Ho, A. E. Ruehli, and P. A. Brennen, "The modified nodal approach to network analysis," IEEE Trans. Circuits Syst., vol. CAS-22, pp. 504-509, 1975.

[20] D. Kincaird and W. Cheney, Numerical Analysis. Pacific Grove, CA: Brooks/Cole, 1991, ch. 4.

[21] J. R. Bunch and D. J. Rose, Eds., Sparse Matrix Computations. New York: Academic, 1976.

[22] R. A. Saleh and A. R. Newton, Mixed-Mode Simulation. Norwell, MA: Kluwer, 1990, ch. 3.

[23] K. G. Nichols, T. J. Kazmierski, M. Zwolinski, and A. D. Brown, "Overview of SPICE-like circuit simulation algorithms," Proc. Inst. Electr. Eng., vol. CDS-141, pp. 242-250, 1994.

[24] J. G. Zhu, S. Y. R. Hui, and V. S. Ramsden, "A dynamic equivalent circuit model for solid magnetic cores for high frequency operations," IEEE Trans. Power Electron., vol. 10, pp. 791-795, Nov. 1995.

[25] P. Holmberg, A. Bergqvist, and G. Engdahl, "Modeling eddy currents and hysteresis in a transformer laminate," IEEE Trans. Magn., vol. 33, pp. 1306-1309, Mar. 1997.

[26] J. Roberts, "Analog treatment of eddy currents and magnetic flux penetration in saturated iron," Proc. Inst. Electr. Eng. Part C, vol. 109, pp. 406-411, 1962. 